\title{
Cluster mass profiles from weak lensing: The influence of substructure
}

\author{
L. J. King ${ }^{1,2}$, P. Schneider ${ }^{1}$, and V. Springel ${ }^{2}$ \\ 1 Institut für Astrophysik und Extraterrestrische Forschung der Universität Bonn, Auf dem Hügel 71, Bonn, \\ Germany \\ 2 Max Planck Institut für Astrophysik, Karl-Schwarzschild Str 1, Garching bei München, Germany
}

Received 13 June 2001 / Accepted 22 August 2001

\begin{abstract}
The tidal gravitational field of a cluster distorts the shapes of background galaxies. This signature can be used to place constraints on the mass profile of the cluster, using a maximum likelihood analysis to find the best-fit parameterised model. In Schneider et al. (2000) and King \& Schneider (2001) we investigated how accurately parameters can be obtained, concentrating on smooth profiles. Here, we consider a realistic model for substructure in a cluster (from $N$-body simulations), in order to see what influence this has on the recovery of parameters. We find that for a typical cluster at $z_{\mathrm{d}}=0.2$, the presence of substructure increases the error on parameters recovered from weak lensing data by $\approx 3 \%$. Even doubling the substructure amplitude induces only a $\approx 10 \%$ increase in the parameter dispersion: this bodes well for our method.
\end{abstract}

Key words. dark matter - gravitational lensing - large-scale structure of Universe - galaxies: clusters: general methods: statistical

\section{Introduction}

When a cluster acts as a weak gravitational lens, its tidal gravitational field distorts the shapes of background galaxies (shear effect), and its magnification effect changes their observed number density. These imprints on the background galaxy population can be used to constrain the projected mass distribution of the cluster.

The mass distribution of a cluster can be reconstructed in a parameter-free way, enabling individual over-densities to be identified. This method was first realised and developed by Kaiser \& Squires (1993), and has since been applied to many clusters (e.g. Seitz et al. 1996; Fischer \& Tyson 1997; Clowe et al. 1998; Hoekstra et al. 1998; Clowe et al. 2000; Hoekstra et al. 2000). However, deriving parameterised cluster models from weak lensing data is essential when statistical comparisons of clusters are to be made. Furthermore, the error properties of parameterised models are well understood in constrast to those of non-parametric mass maps.

In Schneider et al. (2000; hereafter SKE) we investigated parameterised power-law models for clusters, and developed likelihood techniques to quantify the accuracy with which parameters can be recovered using the magnification and shear information. This work was extended in King \& Schneider (2001; hereafter KS) to encompass

Send offprint requests to: L. J. King,

e-mail: lindsay@astro.uni-bonn.de
NFW (Navarro et al. 1996, 1997) and ellipsoidal models (Kormann et al. 1994), and the effect of uncertainty in the redshifts of potential source galaxies was examined. In general, since the shear information places tighter constraints on the radial mass profile, this was our main focus.

A parameterised model, such as the NFW profile, describes a cluster's azimuthally averaged and radially smoothed projected mass distribution. A preliminary consideration of how substructure influences the characteristic errors on recovered parameters was described in KS, and a study of more realistic substructure is presented here, using a cluster derived from the $N$-body simulations of Springel et al. (2001). We also increase the amplitude of the substructure in the cluster simulation so as to examine how our shear likelihood analysis performs. Further, we ask how the ability to distinguish between different families of parameterised models (namely the NFW and general power-law model) is effected by the presence of substructure.

The structure of this paper is as follows: the notation and lensing relationships used are outlined in Sect. 2. In Sect. 3 we summarise the basis of using lensed galaxy ellipticities to constrain a cluster's mass profile, and how the shear information is incorporated into a likelihood analysis. The model used for substructure and the smooth parameterised models used during parameter recovery are described in Sect. 4. Our results are presented in Sect. 5, and we finish with a discussion and conclusions in Sect. 6 . 


\section{Notation and basis of the shear method}

In this section the notation and basic relationships used throughout this paper are introduced. Throughout, standard lensing notation is used (e.g. Schneider et al. 1992; Bartelmann \& Schneider 2001).

The surface mass density of a lens at position $\boldsymbol{\theta}$ is denoted by $\Sigma(\boldsymbol{\theta})$ and the critical surface mass density of a lens at redshift $z_{\mathrm{d}}$ for sources at redshift $z$ by $\Sigma_{\text {crit }}(z) \equiv$ $\frac{c^{2}}{4 \pi G} \frac{D_{\mathrm{s}}}{D_{\mathrm{d}} D_{\mathrm{ds}}}$, where $D_{\mathrm{s}}, D_{\mathrm{d}}, D_{\mathrm{ds}}$ are the observer-source, observer-lens and lens-source angular diameter distances respectively. The dimensionless surface mass density of a lens, $\kappa(\boldsymbol{\theta}, z)$, is the ratio of $\Sigma / \Sigma_{\text {crit }}$, and a Poisson-like equation relates the deflection potential $\psi(\boldsymbol{\theta})$ to $\kappa$

$\nabla^{2} \psi=2 \kappa$.

The complex shear is a combination of second derivatives of the potential

$\gamma=\gamma_{1}+\mathrm{i} \gamma_{2}=\left(\psi_{11}-\psi_{22}\right) / 2+\mathrm{i} \psi_{12}$

(the subscript indices denote partial derivatives with respect to the position $\boldsymbol{\theta}$ on the sky). Further, $g=\gamma /(1-\kappa)$ is the complex reduced shear. The magnification of an image is the inverse of the Jacobian determinant of the lens equation,

$\mu(\boldsymbol{\theta})=[\operatorname{det} \mathcal{A}(\boldsymbol{\theta})]^{-1} ; \quad \operatorname{det} \mathcal{A}=(1-\kappa)^{2}-|\gamma|^{2}$.

In KS we adopted a toy model to describe the substructure in a lens, so the shear field (and hence the resulting image ellipticities) was readily obtained. Since simulations are used to obtain a map of $\kappa$ for a cluster here, another method must be used to derive the shear field. Combining the Fourier transforms of Eqs. (1) and (2) gives an elegant relationship between $\kappa$ and the components of $\gamma$ in Fourier transform space (Kaiser \& Squires 1993)

$\tilde{\gamma}=\frac{\left(k_{1}^{2}-k_{2}^{2}\right) \tilde{\kappa}+2 k_{1} k_{2} \tilde{\kappa}}{k_{1}^{2}+k_{2}^{2}}$,

where $\boldsymbol{k}$ is the usual wave vector.

Throughout this paper, to highlight the influence of substructure, we consider fairly low redshift clusters $\left(z_{\mathrm{d}}=0.2\right)$, where the redshift distribution of the source galaxy population can safely be neglected. This amounts to approximating them to be located at a redshift corresponding to the mean value of their lensing effectiveness parameter $w(z)$, which is taken to be $z=1$. If the cluster is at a higher redshift, $\gtrsim 0.25$ say, then the redshift distribution of the galaxies becomes important and this sheet approximation is no longer robust (Bartelmann \& Schneider 2001). Another motivation for considering $z_{\mathrm{d}}=0.2$ clusters is that this is a relevant redshift for wide field imaging studies - for instance Abell 1689 at $z_{\mathrm{d}}=0.18$ (see Clowe $\&$ Schneider 2001 for details of the observations, and King et al. 2001 for application of the shear method). Further, we restrict our attention to rather well evolved, "normal" clusters - for instance constraining the mass distribution of a "multiple core" cluster such as Abell 370 would probably be inappropriate for this technique.
For consistency with the $N$-body simulations used, a $\Lambda \mathrm{CDM}$ cosmology in which $\Omega=0.3, \Lambda=0.7$ and $H_{0}=$ $70 \mathrm{~km} \mathrm{~s}^{-1} \mathrm{Mpc}^{-1}$ is adopted. In this case, the relevant angular diameter distances can be determined using the relationships given in Bartelmann \& Schneider (2001).

\section{Using galaxy ellipticities to constrain mass profiles}

We begin this section by giving the basis of the shear method, and then summarise the likelihood treatment that uses the shear information.

\subsection{Basis of the shear method}

The ellipticity of a galaxy $\epsilon$ is a complex number whose modulus is $|\epsilon|=(1-r) /(1+r)$, in the case of elliptical isophotes with axis ratio $r \leq 1$, and whose phase is twice the position angle of its major axis. The ellipticity probability distribution is a Gaussian distribution with dispersion $\sigma_{\epsilon^{\mathrm{s}}}$

$p_{\epsilon^{\mathrm{s}}}=\frac{\exp \left(-\left|\epsilon^{\mathrm{s}}\right|^{2} / \sigma_{\epsilon^{\mathrm{s}}}^{2}\right)}{\pi \sigma_{\epsilon^{\mathrm{s}}}^{2}\left[1-\exp \left(-1 / \sigma_{\epsilon^{\mathrm{s}}}^{2}\right)\right]}$.

The tidal gravitational field of a lens changes an individual source ellipticity $\left(\epsilon^{\mathrm{s}}\right)$ into an image ellipticity $\epsilon$. We concentrate on the non-critical regime $(\operatorname{det} \mathcal{A}>0$ ) where this transformation is

$\epsilon=\frac{\epsilon^{\mathrm{s}}+g}{1+g^{*} \epsilon^{\mathrm{s}}}$

where the ${ }^{*}$ denotes complex conjugation. In the critical regime $(\operatorname{det} \mathcal{A}<0$ )

$\epsilon=\frac{g \epsilon^{\mathrm{s}^{*}}+1}{g^{*}+\epsilon^{\mathrm{s}^{*}}}$.

The lensed and unlensed probability distributions are related through

$p_{\epsilon}=p_{\epsilon^{\mathrm{s}}}\left|\frac{\mathrm{d}^{2} \epsilon^{\mathrm{s}}}{\mathrm{d}^{2} \epsilon}\right|$,

when the distribution in source redshift is unimportant.

Since the expectation value for the lensed ellipticity $\langle\epsilon\rangle=g$ in the non-critical regime (e.g. Schramm \& Kayser 1995), and $\langle\epsilon\rangle=1 / g^{*}$ in the critical regime (Seitz $\&$ Schneider 1997), the distorted images of background galaxies can be used to constrain the cluster model. This is the basis of the shear method used in SKE, KS and this paper.

\subsection{Likelihood treatment}

Applying likelihood techniques enables the best-fitting parameters and error estimates for simulated or real observations to be obtained (see for example Press et al. 1992). As in SKE and KS, it is more sensible computationally to 
minimise log-likelihood functions to achieve this, since a large number of galaxies is involved.

Galaxy catalogues are lensed by a cluster derived from $N$-body simulations, whose azimuthal $\kappa$ average can be described by a parameterised model, with true parameters $\pi_{\mathrm{t}}$ (please see Sect. 4.3 for details of the model families considered here).

The best-fit parameters obtained after applying the likelihood treatment to the resultant lensed galaxy catalogues are denoted by $\pi_{\max }$, and the trial parameters (en route to minimisation) by $\pi$ throughout. Quantities that refer to the true model value are subscripted with a "t", and those referring to observed values are subscripted with an " $i$ ". We assume that the observations are carried out in an aperture centred on the cluster, with inner radius $\theta_{\text {in }}$ and outer radius $\theta_{\text {out }}$. The cluster centre is fixed from the simulations, although for real observations this would be determined from the data; we discuss this in Sect. 5.4.

Inside this aperture there are $N_{\gamma}$ images at positions $\boldsymbol{\vartheta}_{i}$ for which an ellipticity can be measured (the number density of galaxies that can be used in the shear method is denoted by $n_{\gamma}$ ). There can be an overlap between the two sets of galaxies. The background galaxy number counts locally follow a power law with slope $\beta$, which we take to be 0.5 .

\subsubsection{Likelihood functions}

The shear log-likelihood function and ensemble averaged log-likelihood function are reproduced here; the reader is referred to SKE for the details and derivations. Minimising these functions gives $\pi_{\max }$, the most likely parameters given the observations.

The shear log-likelihood function is

$\ell_{\gamma}=-\sum_{i=1}^{N_{\gamma}} \ln p_{\epsilon}\left(\epsilon_{i} \mid g\left(\boldsymbol{\vartheta}_{i}\right)\right)$

and the noise in this method arises from the intrinsic dispersion in the galaxy ellipticity distribution, $\sigma_{\epsilon^{\mathrm{s}}}$. Equation (9) just depends on how probable particular lensed ellipticities are, given the $\pi$ under consideration. An advantage of using shape information over magnification information is that it does not require the unlensed number density to be accurately known. Equation (9) can be used numerically with the exact probability distribution for $p_{\epsilon}$.

For analytic work the log-likelihood function $\ell_{\gamma}$ can be approximated by

$\ell_{\gamma}=\sum_{i=1}^{N_{\gamma}} \frac{\left(\epsilon_{i}-g\left(\boldsymbol{\vartheta}_{i}\right)\right)^{2}}{\sigma_{\epsilon}^{2}\left(g\left(\boldsymbol{\vartheta}_{i}\right)\right)}+2 \ln \sigma_{\epsilon}\left(g\left(\boldsymbol{\vartheta}_{i}\right)\right)$

where $\sigma_{\epsilon} \approx\left(1-|g|^{2}\right) \sigma_{\epsilon^{\mathrm{s}}}$. For $|g| \leq 1.0$, this approximation is accurate to better than $\approx 5 \%$ when $\sigma_{\epsilon^{\mathrm{s}}}=0.2$ (SKE).

The ensemble averaged log-likelihood functions allow the characteristic parameter errors to be derived. For the

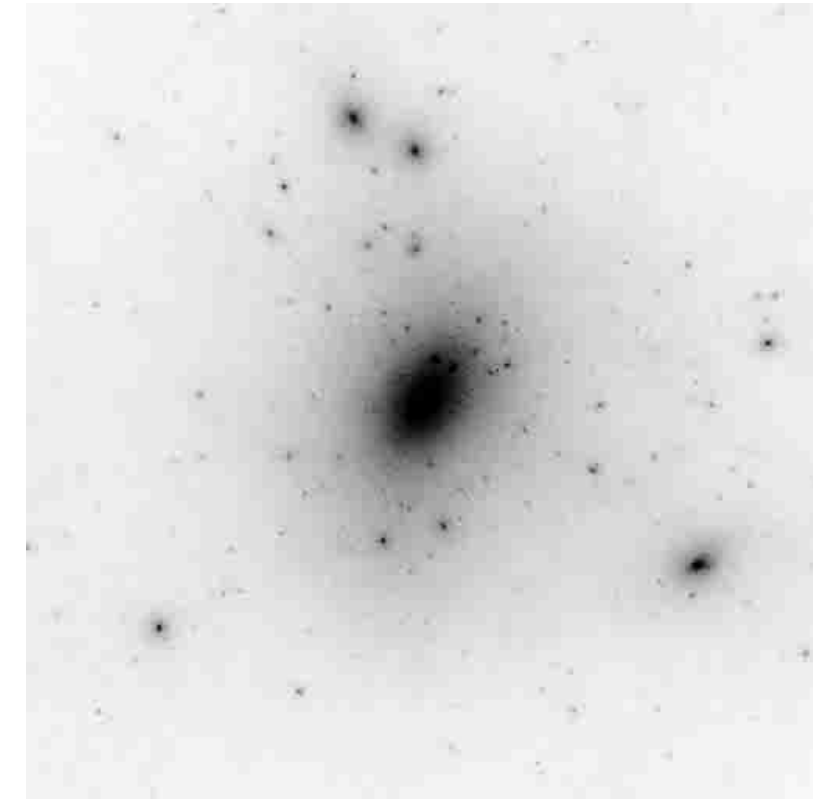

Fig. 1. This figure shows the dimensionless surface mass density of a cluster taken from the $N$-body simulations of Springel et al. (2001), scaled down so that $\kappa \approx 2$ at its centre.

shear we have shown in SKE that

$$
\begin{aligned}
\left\langle\ell_{\gamma}\right\rangle= & n_{\gamma} \int \mathrm{d}^{2} \vartheta\left[\mu_{\mathrm{t}}(\boldsymbol{\vartheta})\right]^{\beta-1} \\
& \times\left(\frac{\left|g(\boldsymbol{\vartheta})-g_{\mathrm{t}}(\boldsymbol{\vartheta})\right|^{2}+\sigma_{\epsilon, \mathrm{t}}^{2}(\boldsymbol{\vartheta})}{\sigma_{\epsilon}^{2}(\boldsymbol{\vartheta})}+2 \ln \sigma_{\epsilon}(\boldsymbol{\vartheta})\right),
\end{aligned}
$$

where again $\mu_{\mathrm{t}}, g_{\mathrm{t}}$ and $\sigma_{\epsilon, \mathrm{t}}$ are determined for $\pi_{\mathrm{t}}$.

\section{Lens models and Monte-Carlo simulations of lensing}

Now we describe the model used for substructure, and how the Monte-Carlo simulations of lensing were carried out.

\subsection{Substructure model}

In KS we considered a toy model for substructure, composed of a smooth NFW profile with additional (net mass zero "Mexican hat") equal amplitude sub-clumps. Here we adopt a cluster taken from the high resolution $N$-body simulations of Springel et al. (2001), for a more realistic description of substructure. The cluster we work with was simulated in a $\Lambda$ CDM cosmology $\left(\Omega_{0}=0.3, \Omega_{\Lambda}=0.7\right.$, $\Gamma=0.21, \sigma_{8}=0.9$ and $\left.H_{0}=70 \mathrm{~km} \mathrm{~s}^{-1} \mathrm{Mpc}^{-1}\right)$. In the $\mathrm{S} 4$ simulation which we use, the particle mass was $4.68 \times 10^{7} h^{-1} M_{\odot}$ and the resolution (i.e. gravitational softening length) $0.7 h^{-1} \mathrm{kpc}$. The cluster is modelled with almost 20 million particles, in which $\approx 4800$ substructures can be resolved, comprising $\approx 12 \%$ of its total mass.

Our primary focus is the surface mass density ( $\kappa$ map) obtained by directly projecting the particles in a cube of side length $4 \mathrm{Mpc}$ centred on the cluster onto a grid on 


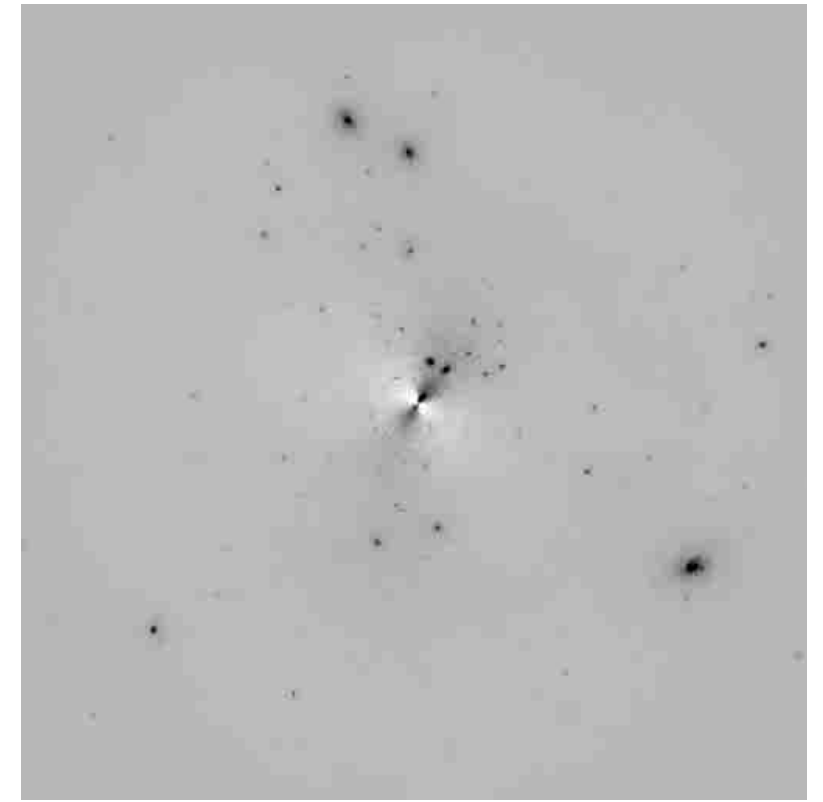

Fig. 2. This figure shows $\kappa_{\text {sub }}$, the difference between the original $\kappa$ map $\left(\kappa_{\text {tot }}\right)$ and the azimuthally averaged $\kappa$ map $\left(\kappa_{\text {smo }}\right)$.

the $x-y$ face. The projection process is referred to as NGP (nearest gridpoint) assignment. Because of the large number of particles involved, no further smoothing was necessary.

We then scaled the surface mass density so that $\kappa \sim 2$ at the centre. Figure 1 shows $\kappa$ for the cluster. In order to check whether our results are influenced by the specific choice of projection axis, we also projected the same cube onto the $y-z$ face. We come back to this projection in Sect. 5.

Taking the $\kappa$ map, which we refer to below as $\kappa_{\text {tot }}$, and performing an azimuthal average around the cluster centre results in the map $\kappa_{\text {smo }}$.

The difference between the smoothed and nonsmoothed maps gives the substructure, $\kappa_{\text {sub }}$, which is shown in Fig. 2. In order to investigate different levels of substructure, while keeping the mass of the cluster fixed, $\kappa_{\text {sub }}$ can be scaled and added to $\kappa_{\text {smo }}$.

\subsection{Monte-Carlo simulations of lensing}

The shear map corresponding to the $\kappa$ map of the simulated cluster was obtained using Fourier transform methods implemented in routines in Nick Kaiser's IMCAT software (http://www.ifa.hawaii.edu/ kaiser/imcat/).

We assume that the data field is 30 '0 on a side, which is approximately the same as the WFI (Wide Field Imager) on the ESO 2.2 m telescope on La Silla. The number density of background galaxies for which an ellipticity can be measured, and can therefore be used for the shear method, is $n_{\gamma}$. Unless otherwise stated, $n_{\gamma}=30 \operatorname{arcmin}^{-2}$ (the typical number density used for shear analysis of deep groundbased data). The expected number of galaxies in the aperture $\langle N\rangle$ is determined, and a random deviate drawn from a Poisson distribution of mean $\langle N\rangle$ gives the number of galaxies in the unlensed galaxy catalogue $\langle N\rangle_{\mathrm{P}}$. These galaxies are randomly distributed and individual galaxy ellipticities are drawn from a Gaussian probability distribution with 2-D dispersion $\sigma_{\epsilon^{\mathrm{s}}}=0.2$ (see Eq. (5)). At this stage, the catalogue of unlensed galaxies contains random $\boldsymbol{\theta}_{i}$ and $\epsilon_{i}^{\mathrm{s}}$. For a given cluster simulation, with parameters $\pi_{\mathrm{t}}$, the lensed ellipticities for each galaxy $\epsilon_{i}$ are obtained using the relationship (6). To account for magnification, a fraction of the sources is rejected: if a uniform random deviate $[0,1]$ is larger than $\left[\mu_{\mathrm{t}}\left(\pi_{\mathrm{t}}, \boldsymbol{\theta}_{i}\right)\right]^{\beta-1}$, the galaxy is excluded from the catalogue. Note that this means that more highly magnified sources are more likely to be rejected at this stage - far away from the cluster centre, when $\mu_{\mathrm{t}} \rightarrow 1$, the lensed and unlensed number densities must be equivalent.

Finally, the catalogue contains $\boldsymbol{\theta}_{i}$ and $\epsilon_{i}$ for each lensed galaxy, representing a single lensed data set. The loglikelihood functions are then minimised to obtain $\pi_{\max }$ for the catalogues, for a particular family of lens models whose properites are outlined in the next subsection.

\subsection{Profiles}

The azimuthal average of $\kappa$ for the simulated cluster can be described by a parameterised model. Our main emphasis is on the NFW profile, and we also briefly consider the isothermal and generalised power-law profiles.

\subsubsection{The isothermal profile}

The isothermal profile is characterised by a velocity dispersion that is constant with radius. It is parameterised by an Einstein radius $\theta_{\mathrm{E}}$, which marks the boundary between the strong and weak lensing regimes

$\kappa(\theta)=0.5\left(\frac{\theta}{\theta_{\mathrm{E}}}\right)^{-1}$

The mean surface mass density inside $\theta$ is $\bar{\kappa}(\theta)=2 \kappa(\theta)$, and further $|\gamma|=\kappa$.

Fitting an isothermal profile directly to $\kappa$ for the simulated cluster results in a best-fit $\theta_{\mathrm{E}}=0$ ! 17 , over the radial range 1. to to 10 '0; the fit is shown in Fig. 3 .

\subsubsection{The power-law profile}

A two-parameter model that generalises the isothermal profile can be characterised with a normalisation $b$ and a slope $q$, such that

$\kappa(\theta)=b \theta^{-q}$

$\bar{\kappa}(\theta)=\frac{2 b}{2-q} \theta^{-q}$

and

$\gamma(\theta)=b \theta^{-q}\left(\frac{2}{2-q}-1\right)$ 
Our main purpose in considering this model is for later comparison with a two-parameter NFW model. The bestfit general power-law profile over the radial range 1!.0 to 10.0 is shown in Fig. 3; the corresponding parameters are $b=0.117$ and $q=1.498$. Note that a single slope power-law is a good representation of the profile over the range considered.

\subsubsection{The NFW profile}

The properties of the NFW profile in the context of gravitational lensing have been discussed by authors including Bartelmann (1996) and Wright \& Brainerd (2000). We can parameterise this profile with a virial radius $r_{200}$, and a dimensionless concentration parameter $c$, which are related through a scale radius $r_{\mathrm{s}}=r_{200} / c$. Inside $r_{200}$, the mass density of the halo equals $200 \rho_{\mathrm{c}}$, where $\rho_{\mathrm{c}}=\frac{3 H^{2}(z)}{8 \pi G}$ is the critical density of the Universe at the redshift of the halo. The characteristic overdensity of the halo, $\delta_{\mathrm{c}}$, is related to $c$ through

$\delta_{\mathrm{c}}=\frac{200}{3} \frac{c^{3}}{\ln (1+c)+c /(1+c)}$.

Then the density profile is

$\rho(r)=\frac{\delta_{\mathrm{c}} \rho_{\mathrm{c}}}{\left(r / r_{\mathrm{s}}\right)\left(1+r / r_{\mathrm{s}}\right)^{2}}$,

which is shallower than isothermal $\left(r^{-2}\right)$ near the halo center and steeper than isothermal for $r \gtrsim r_{\mathrm{s}}$. Please see the appendix for the expressions for $\kappa, \bar{\kappa}, \gamma$ and $g$.

The NFW profile that gives the best fit directly to the simulated cluster's $x-y$ projected $\kappa$ map over the range 1.0 to 10.0 has $r_{\mathrm{s}}=0.174 \mathrm{Mpc}$; the fit is shown in Fig. 3. Note that the NFW profile gives a more reasonable direct fit to the simulation than the isothermal profile, but a comparable fit to the power-law model. At smaller radii, the simulation profile deviates from an NFW profile. The best-fit to the $y-z$ projected $\kappa$ map has $r_{\mathrm{s}}=0.199 \mathrm{Mpc}$.

\section{Results}

\subsection{How does substructure amplitude effect the shear method?}

The first cluster lens we consider is the original simulation, projected onto the $x-y$ plane, without any modification of the substructure amplitude.

We refer to this model as $\mathcal{M}_{1}$. Catalogues of lensed galaxies were generated using the techniques described above, randomising the positions and intrinsic ellipticities of the unlensed galaxies prior to the creation of each new catalogue. The best-fit NFW models were obtained in an aperture centred on the cluster $\left(\theta_{\text {in }}=1 ! 0\right.$ and $\left.\theta_{\text {out }}=10^{\prime} 0\right)$ for 500 catalogues of lensed galaxies. The same procedure was repeated for the azimuthally averaged cluster model $\mathcal{M}_{\text {smo }}$. The amplitude of the substructure was scaled by a factor of two (model $\mathcal{M}_{2}$ ), and the bestfit models again obtained for the same 500 catalogues of

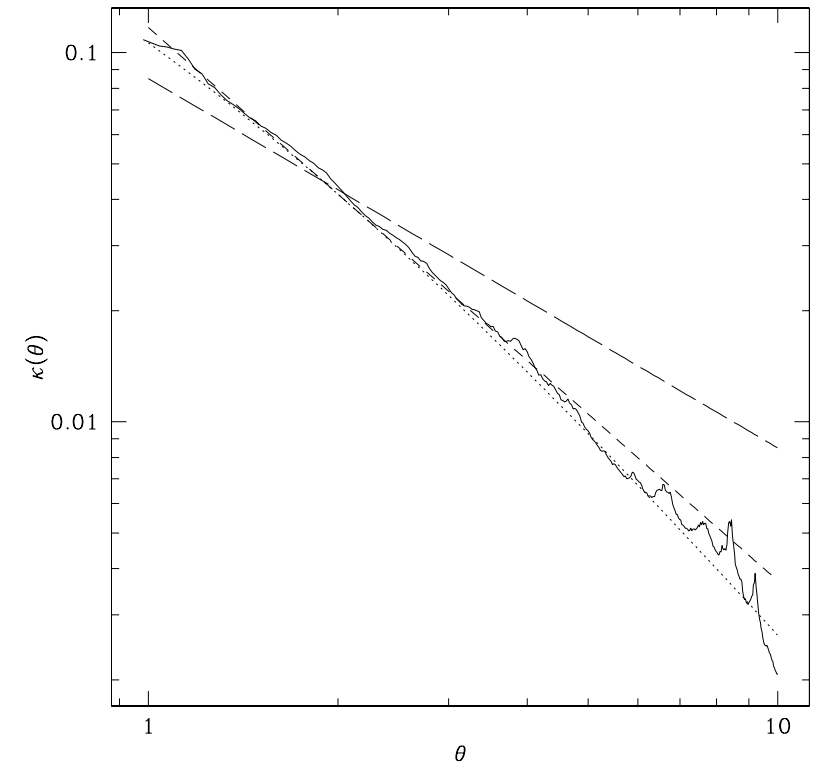

Fig. 3. This figure shows the azimuthally averaged dimensionless surface mass density $\left(\kappa_{\mathrm{smo}}\right)$ for the simulated cluster (solid line), along with the best-fit NFW projection (short dash line), single slope power-law (medium dash line) and SIS (long dash line) profiles between 1.0 and 10.0 .

lensed galaxies. This serves to illustrate the effect of extreme substructure fluctuations in clusters at this redshift.

For recovery with the NFW model, the dispersion in $r_{\mathrm{s}}$ is $\sigma_{r_{\mathrm{s}}}=0.064(0.066,0.069) \mathrm{Mpc}$ for $\mathcal{M}_{\text {smo }}\left(\mathcal{M}_{1}, \mathcal{M}_{2}\right)$. The distribution of $r_{\mathrm{s}}$ for the best-fit NFW models for $\mathcal{M}_{1}$ and $\mathcal{M}_{2}$ is shown in Fig. 4. There is a small offset between the mean value of the recovered parameters which is most likely attributable to local magnification and shear effects (recall from Sect. 4.2 that we allow for magnification in that a certain fraction of sources are rejected). With increasing substructure amplitude, the magnification distribution of background galaxies is broadened by local over- and under-densities, and there is a small net decrease in the average reduced shear of a galaxy lensed by the cluster.

It should be noted that the dispersion in parameters induced by increasing the intrinsic ellipticity distribution dispersion to $\sigma_{\epsilon^{\mathrm{s}}}=0.3$, and considering lensing through $\mathcal{M}_{\mathrm{smo}}$, gives rise to a larger dispersion in $r_{\mathrm{s}}\left(\sigma_{r_{\mathrm{s}}}=\right.$ $0.095 \mathrm{Mpc}$ ) than that obtained by lensing through $\mathcal{M}_{2}$.

\subsection{How easily can profile families be distinguished?}

The best-fit isothermal models and power-law models were then obtained for $\mathcal{M}_{1}$ using the same 500 catalogues of lensed galaxies. Now we ask how likely it is that the best-fit model to the catalogues is NFW as opposed to isothermal or general power-law. Although the vast majority (about $84 \%$ ) of best-fit models are NFW models as opposed to isothermal models, the differences in their likelihoods is quite small. 
Formally, models with the same number of parameters should be compared, so we consider the more general power-law model. For $\theta_{\text {in }}=1.0$ and $\theta_{\text {out }}=10.0$ the likelihoods of the best-fit NFW and general power-law models reveal that it is statistically impossible to distinguish the profiles, with roughly equal percentages of best-fit models coming from each family $(46.5 \% \mathrm{NFW}$ and $53.5 \%$ powerlaw). From Fig. 3 it is easy to see why this is the case: for the aperture considered, the power-law model also gives a very good fit to the cluster profile.

We decreased $\theta_{\text {in }}$ to 0.5 and repeated the Monte-Carlo simulations through $\mathcal{M}_{1}$, recovering the best-fit NFW and power-law models for 500 catalogues of lensed galaxies. For this aperture, $84 \%$ of the best-fit models are from the NFW family. Figure 5 shows the percentage of catalogues as a function of the difference between the likelihoods of the best-fit NFW and power-law models, $2\left(\ell_{\mathrm{NFW}}-\ell_{\mathrm{POW}}\right)$. Catalogues with negative values of $2 \Delta \ell$ are those where the overall best-fit model is an NFW model. Again, note that the confidence with which the models are discriminated is fairly low.

\subsection{Does the choice of projection axis influence the results?}

If our cluster was perfectly spherical, then the choice of projection axis would not effect the results. In order to check how the scatter of recovered parameters depends on the choice of projection axis, we used the same 500 initial catalogues as above and lensed them through the $y-z$ projection of the cluster. Best-fit NFW models were obtained for the azimuthally averaged and the 2 -D cluster projection with unmodified substructure amplitude, using data from an aperture with $\theta_{\text {in }}=1.0$ and $\theta_{\text {out }}=10$ ' 0 . The dispersions in $r_{\mathrm{s}}$ for these models are $\sigma_{r_{\mathrm{s}}}=0.069$ and $0.072 \mathrm{Mpc}$ respectively (differing by $4 \%$ ). Now consider the difference between $\bar{r}_{\mathrm{s}}\left(\sigma_{r_{\mathrm{s}}}\right)$ recovered from the 2-D $x-y$ and $y-z$ projections: 0.227 (0.066) and $0.218(0.072) \mathrm{Mpc}$. Note that the $\bar{r}_{\mathrm{s}}$ values are well within $\sigma_{r_{\mathrm{s}}}$ and are therefore statistically consistent. We also examined the error on $\sigma_{r_{\mathrm{s}}}$ by bootstrapping, drawing 100000 realisations of 500 best-fit profiles at random from the best-fit profiles to our original 500 catalogues. The corresponding values of $\sigma_{r_{\mathrm{s}}}$ for each set of 500 catalogues and the dispersion in $\sigma_{r_{\mathrm{s}}}$ for the 100000 realisations are $0.066(0.003)$ and $0.072(0.003) \mathrm{Mpc}$ for the $x-y$ and $y-z$ 2 -D projections. The dispersions in $\sigma_{r_{\mathrm{s}}}$ differ by more than $1-\sigma$.

The projection effect just described above arises from asphericity in the cluster itself, since the simulation volume considered here localises the cluster. However, structures such as filaments may also contribute to the projected surface mass density, and to the lensing signal. Using clusters from a large scale cosmological simulation, Metzler et al. (1999) find that positive biases of $\sim 20 \%$ in the inferred cluster mass are typical for a cluster at $z_{\mathrm{d}}=0.5$ (the effect is likely to be smaller at $z_{\mathrm{d}}=0.2$ ).

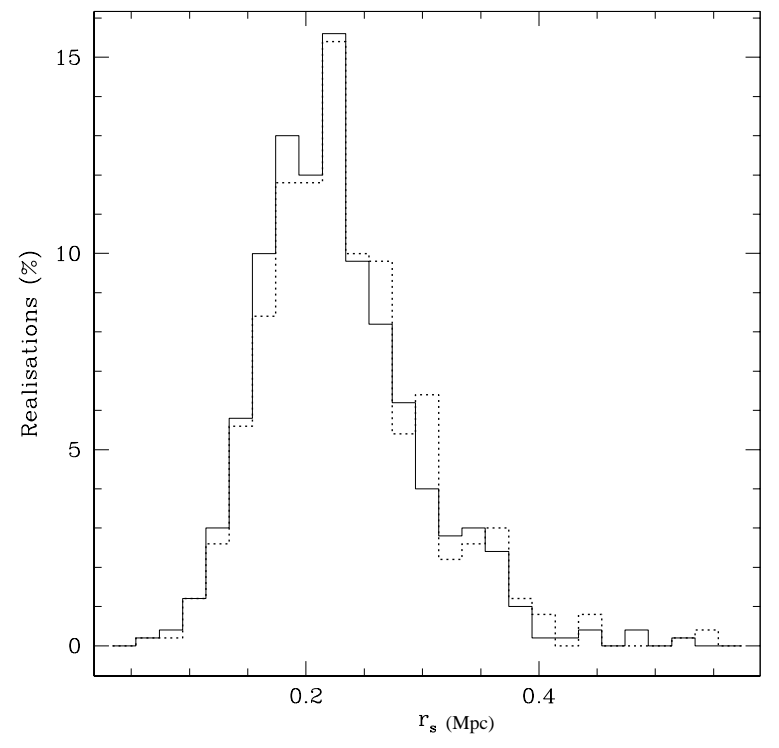

Fig. 4. This figure shows the percentage of realisations as a function of $r_{\mathrm{s}}$, for the best-fit NFW models. The solid line is for Monte-Carlo simulations through $\mathcal{M}_{1}$ and the dashed line for Monte-Carlo simulations through $\mathcal{M}_{2}$.

\subsection{Practical determination of the cluster centre}

In what preceeded, we took a fixed cluster centre from the simulations. In practice, the cluster centre can be determined a priori using the lensing information directly, making no assumption about the profile. Several schemes are described in Schneider \& Seitz (1995). The one we consider now, which they found to be very reliable when the data considered is solely from the weak lensing regime, is to find the position that maximises the sum of the tangential ellipticities of the lensed galaxies. For the $y-z$ projection described above, we calculated the centre directly using the ellipticities from 500 Monte Carlo simulations of lensing. The dispersion in the values of the distance between the recovered cluster centre and the true cluster centre, $\sigma_{\text {centre }}=0.09$, corresponds to a very small deviation in $\Delta r_{\mathrm{s}} \sim 4 \%$. In summary, cluster centres can be determined to such an accuracy as to only marginally change the recovered parameters.

\section{Discussion and conclusions}

In SKE and in KS we performed Monte-Carlo simulations of lensing through a smooth, parameterised cluster model. We briefly touched on the issue of substructure (working with a simple toy model), and in this paper we have used a cluster from numerical simulations as our model. This enables us to investigate how our shear method is effected when a realistic amount of substructure is present.

Our results show that the dispersion of recovered parameters, or the characteristic error expected for a single data set, becomes larger with increasing substructure amplitude. But even when the amplitude of substructure in a realistic cluster is increased by a factor of two, the parameter dispersion is increased by less than $10 \%$. 


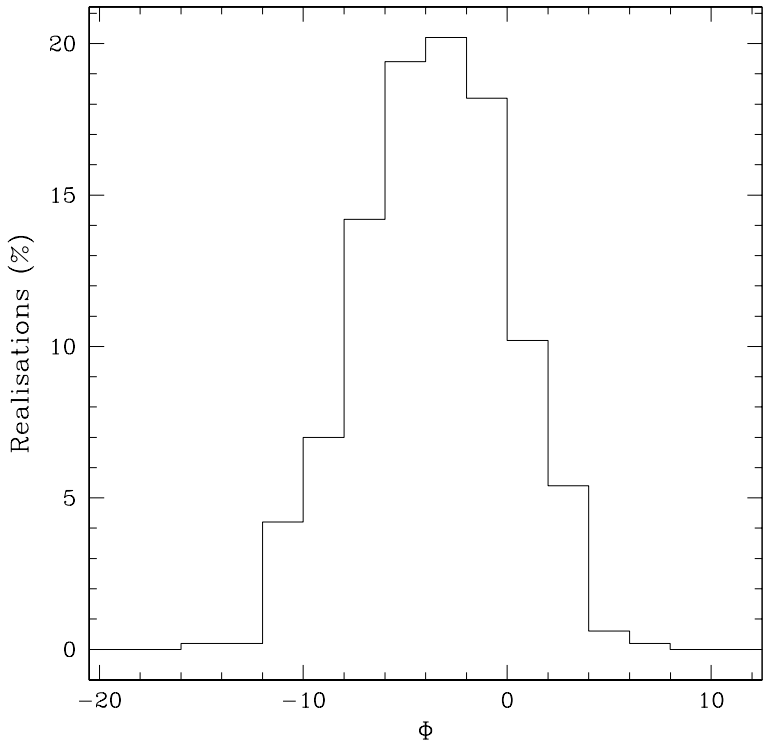

Fig. 5. This figure shows the percentage of realisations as a function of $\Phi=2 \Delta \ell$, where $\Delta \ell$ is the difference in the likelihoods of the best-fit NFW and isothermal models $\ell_{\mathrm{NFW}}-\ell_{\mathrm{POW}}$. Recovery was made in an aperture with $\theta_{\text {in }}=0.5$ and $\theta_{\text {out }}=10^{\prime} .0$.

We concentrated on the $x-y$ projection of the cluster, but we also find that the mean recovered value of $r_{\mathrm{s}}$ is consistent with that of the $y-z$ projection. That the value of the scatter $\sigma_{r_{\mathrm{s}}}$ is $9 \%$ larger for the $y-z$ projection, corresponds to a greater characteristic error for this projection. For a given profile family, a cluster with a larger mass has smaller characteristic errors on parameters derived from weak lensing data: since the mass $M \propto r_{200}^{3}$, then $\Delta M / M \propto r_{200}^{-1}$. Although the mean best-fit values of $r_{200}$ for the $x-y$ and $y-z$ projections are within $1-\sigma$ of each other at $1.114(0.051)$ and 1.066 (0.053) Mpc respectively, the slightly larger value for the $x-y$ projection implies a slightly higher best-fit mass, and tighter ensemble averaged likelihood contours or characteristic errors.

We found a small offset in the recovered parameters when substructure is present, or its amplitude is increased, which is most likely attributable to the local effects of overand under-densities on the ellipticities and magnifications of background galaxies.

For the cluster in question, with the assumed number density of background sources (30 $\operatorname{arcmin}^{-2}$, typical of ground-based observations), and when $\theta_{\text {in }}=1 ! 0$, it is statistically impossible to say whether the best-fit model to the weak lensing data is an NFW profile or a general single slope power-law model. When $\theta_{\text {in }}$ is decreased to 0.5 , the discrimination between models becomes easier since we probe further into the regime below $r_{\mathrm{s}} \sim 1.4$ around which the NFW profile slope changes from isothermal to flatter than isothermal; the single power-law slope cannot provide such a good fit to the data. As we discuss in KS, differentiation between profile families is further facilitated by having space-based or very deep ground-based observations. Another possibility is the stacking of many such clusters - as done for galaxy groups by Hoekstra et al. (2001). A complimentary approach to the classification of cluster profiles using weak lensing data is presented in Bartelmann et al. (2001). This is a statistical method, utilising the $M_{\text {ap }}$ statistics developed in Schneider (1996).

In conclusion, we find that the presence of realistic substructure does not have a serious impact on the shear method developed in SKE and KS.

Acknowledgements. We would like to thank Douglas Clowe and Matthias Bartelmann for very interesting discussions, and Nick Kaiser for providing his IMCAT software. We also thank Matthias for a very careful reading of the manuscript, and an anoymous referee for their helpful comments. This work was supported by the TMR Network "Gravitational Lensing: New Constraints on Cosmology and the Distribution of Dark Matter" of the EC under contract No. ERBFMRX-CT97-0172.

\section{Appendix A: Relationships for NFW Profile}

The radial dependence of the dimensionless surface mass density as a function of a dimensionless radial coordinate $x:=r / r_{\mathrm{s}}$ is given by:

$$
\kappa(x)=\kappa_{k} f(x)
$$

where

$$
\begin{aligned}
& f(x<1)=\frac{1}{x^{2}-1}\left(1-\frac{2 \operatorname{atanh} \sqrt{\frac{1-x}{1+x}}}{\sqrt{1-x^{2}}}\right) \\
& f(x=1)=\frac{1}{3} \\
& f(x>1)=\frac{1}{x^{2}-1}\left(1-\frac{2 \operatorname{atan} \sqrt{\frac{x-1}{1+x}}}{\sqrt{x^{2}-1}}\right)
\end{aligned}
$$

and

$\kappa_{k}=\frac{2 r_{\mathrm{s}} \delta_{\mathrm{c}} \rho_{\mathrm{c}}}{\Sigma_{\mathrm{c}}}$

The mean dimensionless surface mass density inside radius $x$ is:

$\bar{\kappa}(x)=\kappa_{k} h(x)$,

where

$h(x<1)=\frac{2}{x^{2}}\left(\frac{2 \operatorname{atanh} \sqrt{\frac{1-x}{1+x}}}{\sqrt{1-x^{2}}}+\ln \left(\frac{x}{2}\right)\right)$
$h(x=1)=2+2 \ln \left(\frac{1}{2}\right)$
$h(x>1)=\frac{2}{x^{2}}\left(\frac{2 \operatorname{atan} \sqrt{\frac{x-1}{1+x}}}{\sqrt{x^{2}-1}}+\ln \left(\frac{x}{2}\right)\right)$.

The shear $\gamma=\bar{\kappa}-\kappa$ at position $x$ is:

$\gamma(x)=\kappa_{k} j(x)$, 
where

$$
\begin{aligned}
j(x<1)= & \frac{4 \operatorname{atanh} \sqrt{\frac{1-x}{1+x}}}{x^{2} \sqrt{1-x^{2}}}+\frac{2 \ln \left(\frac{x}{2}\right)}{x^{2}}-\frac{1}{x^{2}-1} \\
& +\frac{2 \operatorname{atanh} \sqrt{\frac{1-x}{1+x}}}{\left(x^{2}-1\right) \sqrt{1-x^{2}}} \\
j(x=1)= & 2 \ln \left(\frac{1}{2}\right)+\frac{5}{3} \\
j(x>1)= & \frac{4 \operatorname{atan} \sqrt{\frac{x-1}{1+x}}}{x^{2} \sqrt{x^{2}-1}}+\frac{2 \ln \left(\frac{x}{2}\right)}{x^{2}}-\frac{1}{x^{2}-1} \\
& +\frac{2 \operatorname{atan} \sqrt{\frac{x-1}{1+x}}}{\left(x^{2}-1\right)^{\frac{3}{2}}},
\end{aligned}
$$

and the reduced shear $g=\frac{\gamma}{1-\kappa}$.

\section{References}

Bartelmann, M., \& Schneider, P. 2001, Phys. Rep., 340, 291

Bartelmann, M., King, L. J., \& Schneider, P. 1999, A\&A, in press [astro-ph/0103465]

Broadhurst, T. J., Taylor, A. N., \& Peacock, J. A. 1995, ApJ, 438,49

Clowe, D., Luppino, G. A., Kaiser, N., Henry, J. P., \& Gioia, I. 1998, ApJ, 497, L61

Clowe, D., Luppino, G. A., Kaiser, N., \& Gioia, I. M. 2000, ApJ, 539, 540
Clowe, D., \& Schneider, P. 2001, A\&A, submitted

Fischer, P., \& Tyson, A. J. 1997, AJ, 114, 14

Hoekstra, H., Franx, M., Kuijken, K., \& Squires, G. 1998, ApJ, 504,636

Hoekstra, H., et al. 2001, ApJ, 548, L5

Hoekstra, H., Franx, M., \& Kuijken, K. 2000, ApJ, 532, 88

Kaiser, N., \& Squires, G. 1993, ApJ, 404, 441

King, L. J., \& Schneider, P. 2001, A\&A, 369, 1

King, L. J., Clowe, D., \& Schneider, P. 2001, A\&A, submitted

Kormann, R., Schneider, P., \& Bartelmann, M. 1994, A\&A, 284,285

Metzler, C. A., White, M., Norman, M., \& Loken, C. 1999, ApJ, 520, L9

Navarro, J. F., Frenk, C. S., \& White, S. D. M. 1996, ApJ, 462,563

Navarro, J. F., Frenk, C. S., \& White, S. D. M. 1997, ApJ, 490,493

Press, W. H., Teukolsky, S. A., Vetterling, W. T., \& Flannery, B. P. 1992, in Numerical recipes in FORTRAN, The art of scientific computing, 2nd Ed. (Cambridge: University Press)

Schneider, P., Ehlers, J., \& Falco, E. E. 1992, Gravitational lenses (Springer: New York) (SEF)

Schneider, P. 1996, MNRAS, 283, 837

Schneider, P., King, L. J., \& Erben, T. 2000, A\&A, 353, 41

Schramm, T., \& Kayser, R. 1995, A\&A, 289, L5

Seitz, C., Kneib, J.-P., Schneider, P., \& Seitz, S. 1996, A\&A, 314,707

Seitz, C., \& Schneider, P. 1997, A\&A, 318, 687

Springel, V., White, S. D. M., Tormen, G., \& Kauffmann, G. 2001 [astro-ph/0012055]

Wright, C. O., \& Brainerd, T. G. 2000, ApJ, 534, 34 